\title{
Foreign Bodies - \\ The creation of symbiotic jewellery through the development and application of stimulus-responsive smart materials and microelectromechanical systems
}

\author{
Katharina Vones (MA $\left.{ }^{\text {hons }} B A^{\text {hons }} M A R C A\right)^{1, a}$ \\ AHRC funded Doctoral Researcher
${ }^{1}$ Department of Postgraduate Research, Duncan of Jordanstone College of Art \& Design (DJCAD), The University of Dundee, Perth Road, DD1 4HT, Dundee, Scotland, UK
${ }^{a}$ k.b.childs@dundee.ac.uk

\begin{abstract}
Keywords: smart materials, stimulus-responsive jewellery, contemporary craft, microelectromechanical systems, smart metals, smart polymers, studio jewellery
\end{abstract}

\begin{abstract}
With the increased prevalence of digital technologies in our everyday lives, the questions posed to the contemporary craft practitioner regarding creation of an emotionally resonant interaction between the digitally enhanced object and its wearer have become progressively more prominent in the applied arts. Through examining the notion that human biology is a part of material culture, where the body can be shaped, customised or altered through surgical intervention and scientific innovation, this extended abstract explores how recent developments in material science and wearable technologies can be viewed as contiguous rather than oppositional to the organic processes of the human body and how to bridge the gap between the craft practitioner and scientific discovery. More immediately however, this research project challenges the perception of smart materials and their application within the field of contemporary jewellery in both an artistic and scientific context through proposing the development of symbiotic stimulus-reactive jewellery organisms. Potential practical applications of these jewellery objects exist in the areas of humancomputer interaction, transplant technology, identity management and artificial body modification including prosthetics, where such symbiotic jewellery organisms could be used to develop visually engaging yet multifunctional enhancements of the body.
\end{abstract}

\section{Research Questions}

The following research questions represent the core issues of my practice-led research project that focuses on the development and application of stimulus-responsive smart materials and microelectromechanical systems in the creation of symbiotic jewellery objects:

1) How do we bring material development and wearable technologies into a unified organic whole with each other and with the body?

2) How can the use of stimulus-responsive smart materials enhance the synergy between the wearer's body and the jewellery object?

3) How can the development of 'material enchantment' and 'technological beauty' enhance the use of these materials in the field of contemporary jewellery and thereby strengthen the discipline's capacity to contribute to other spheres and to become instigators for collaborations between the creative arts and sciences? 


\section{Introduction}

Mapping Digital Frontiers With the increasing prevalence of digital technologies in our everyday lives [1], the questions posed to the contemporary craft practitioner with regards to creating an emotionally resonant interaction between the digitally enhanced object and its wearer have become progressively more prominent in the applied arts. Recent developments in the fields of materials science, interaction design and human computer interaction have contributed to a surge in interest on how to bridge the gap between the craft practitioner and scientific discovery. The work undertaken by academic researchers such as Jayne Wallace [2,3,4], Sarah Kettley [5,6,7] and David Humphrey [8] has laid the foundation for further academic enquiry into the subject and in particular its relationship with contemporary jewellery design. While Kettley's research engages with the social context in which wearable computer-enhanced objects cross the interpretive border between the utilitarian artefact and a conceptually expressive artwork, Wallace focuses on creating jewellery objects that, aside from containing interactive technology, also aim to address aesthetic considerations. She stipulates that through generating beauty and technological enchantment an emotionally meaningful interaction between wearer and jewellery object is facilitated, thus asserting the importance of applying to technologically enhanced objects an aesthetic framework that is firmly rooted within the parameters of the contemporary crafts. Leon Williams' body of work, which focuses on medically assistive jewellery, on the other hand addresses the idea that a combination of sophisticated technology embedded within aesthetically desirable jewellery objects can promote a form of personal empowerment [9]. The aesthetic component of the jewellery object enables its wearer to make use of its function in the public domain, thus facilitating the use of medical products that might otherwise be perceived as socially stigmatising. The intersection between technological refinement, the exploration of smart materials and new manufacturing technologies as well as the development of an aesthetic expression that supersedes ideas of mere gadgetry is a recurring theme in this area of research and one which I would like to address with my contribution to the field. Taking Wallace's provision for the amalgamation of technological enchantment and aesthetic beauty [10] as a starting point, my research addresses aesthetic considerations alongside functionality, thus developing material and technological solutions that constitute an integrated and functional yet unified part of the jewellery object as a whole. While previous projects have placed a strong emphasis on simply creating receptacles to accommodate electronic components within a jewellery object [11], the possibilities offered by digital manufacturing technologies such as rapid-prototyping and computer aided design have expanded the aesthetic vocabulary available to the practitioner. Furthermore, the discovery and increasing availability of a range of exciting stimulus-reactive smart materials, such as shape memory alloys $[12,13]$ and thermo- and photo-reactive polymers [14,15] or ceramics, [16] in addition to the progressive miniaturisation of electromechanical components, has turned the prospect of developing jewellery objects that appear to be responsive to their environment, yet depend closely on an interaction with the physiology of the wearer's body to stimulate these responses, from a distant imagining into a feasible goal.

Creating Meaningful Interactions. While the development of smart textiles has generated a significant amount of research activity both on a national and international level, the field of contemporary jewellery has yet to explore the full potential of these newly available materials and technologies. Early propositions for an engagement between smart materials and ornament have been stipulated by researchers such as Professor Norman Cherry, who convincingly argues the possibilities offered by tissue engineering to grow decorative implants from cultured human cells [17]. While the use of smart materials in such an experimental yet theory-based context may have piqued the interest of the field, the common perception of smart materials prevalent within the contemporary jewellery community speaks of a reluctance to perceive these materials as a vehicle for artistic self-expression. In the discipline of fashion design on the other hand, designers such as Hussein Chalayan (Fig.1) and Nancy Tilbury (Fig.2) are successfully combining wearable 


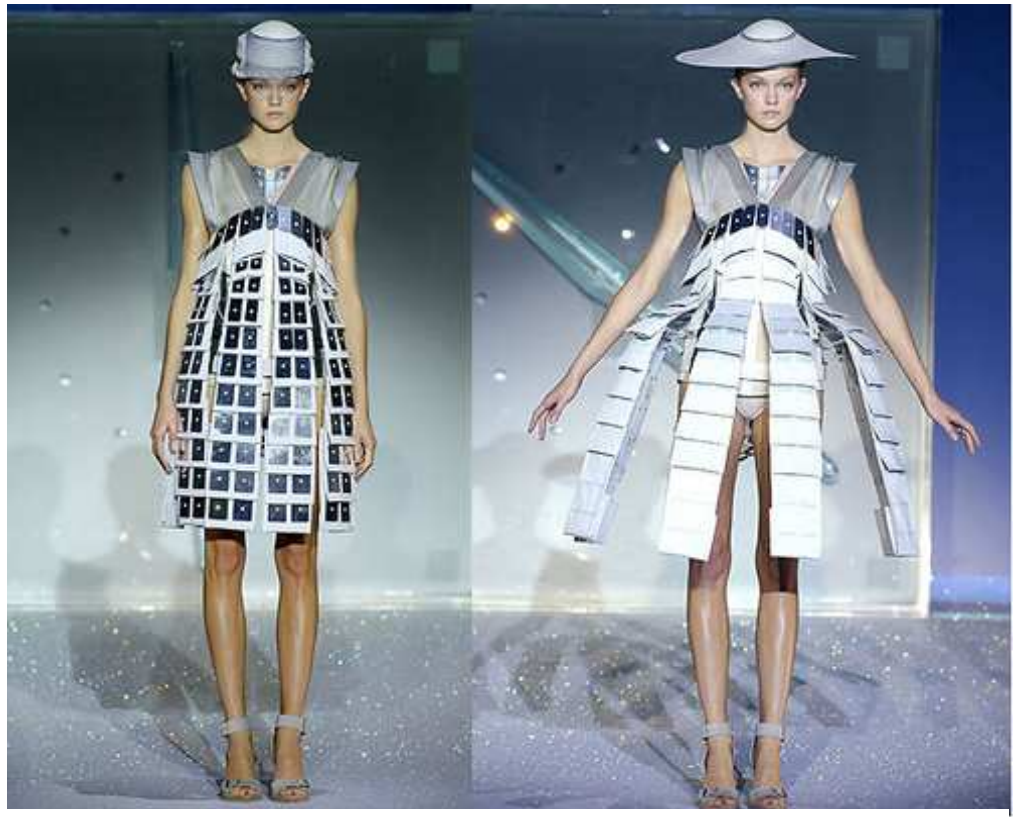

Fig.1: Transform Dress, Hussein Chalayan 2007, SS One Hundred and Eleven Collection technologies and smart materials to create garments that react closely to the physical states of their wearer's bodies. Working methodologies geared towards establishing teams of carefully selected creatives each with their own field of expertise under the supervision of a head designer - a common practice in the fashion industry - working together towards the completion of a single project has meant that the creation of such garments has been facilitated to some extent by enlisting technical personnel. The barrier for such symbiotic relationships to take effect in the field of contemporary studio jewellery is one of process rather than intent - artistic collaborations in this field are uncommon and only too

often the practice of the artist-maker sequestered away in their studio prevails. When such collaborative efforts do occur - such as the Biojewellery Project led by jewellery designers Tobie Kerridge and Nikki Stott in conjunction with bioengineer Ian Thompson - they provide valuable insights and new working practices for all parties involved [18].

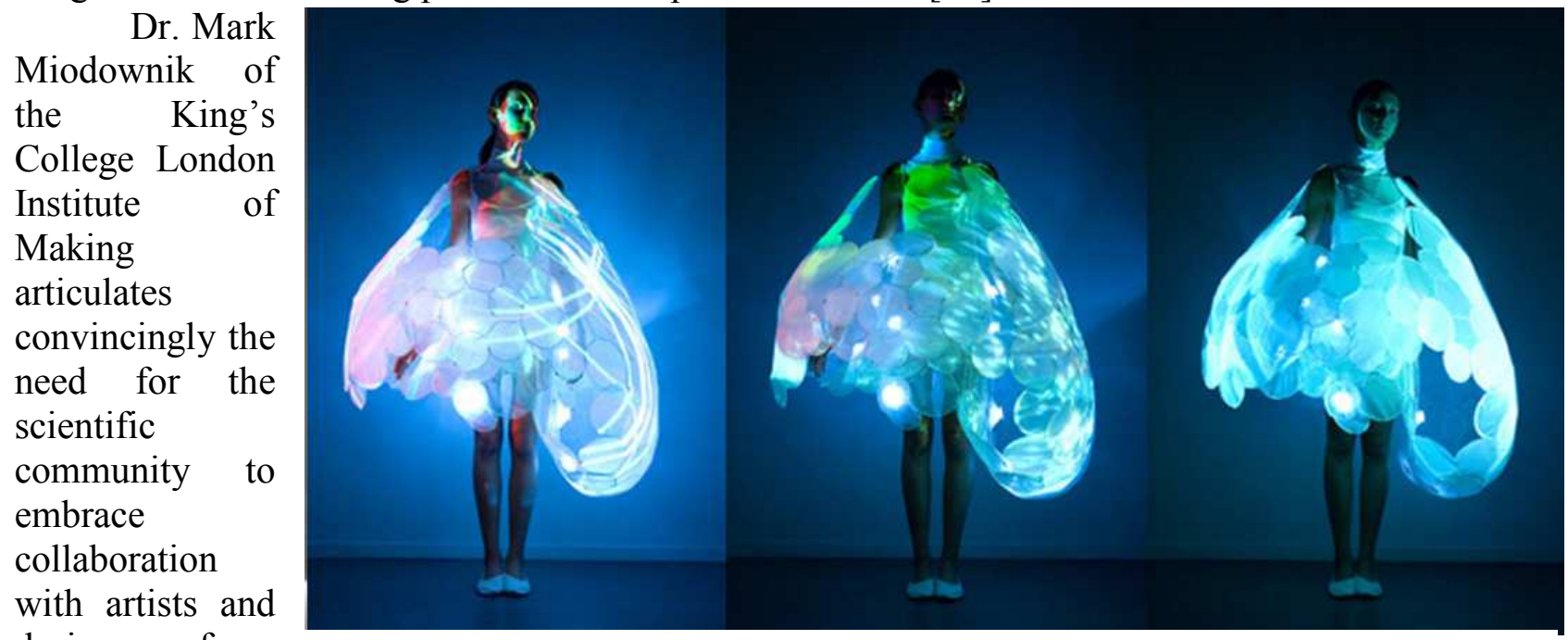
designers from all disciplines in order

Fig.2: Bubelle Dress, Nancy Tilbury et al. 2006, Part of the Phillips SKIN:Dresses Design Probe

facilitate the training of what he terms technical creatives [19]. Such technical creatives, well versed in both scientific and artistic methodologies, will represent a new breed of studio artist, equipped to contribute to the debate surrounding the role of the practitioner in an age defined by digital revolution and material discovery. A growing fascination with and reliance upon technologies and devices that contain some form of interactivity has permeated the cultural fabric of modern society, and my research project provides an essential part of developing a discourse on the place symbiotic jewellery and the contemporary craft practitioner occupy within this setting. The challenge to reconfigure craft-based techniques and aesthetic considerations within a methodological framework focused on the user experience of wearing and interacting with stimulus-reactive jewellery objects will provide the basis for the further development of these ideas in a variety of practical applications. 


\section{Significance}

Towards a unified aesthetic With the increasing prevalence of the notion that human biology is a part of material culture, where the body can be shaped, customised or altered through surgical intervention and scientific innovation, the need arises to explore how recent developments in material science and technological enhancement can be viewed as contiguous rather than oppositional to the organic processes of the human body. Investigating the potential to create a synergetic relationship between the body and objects containing smart materials and wearable technologies, has the potential to both inform and guide these developments. More immediately however, this research project will alter the perception of smart materials and their application within the field of jewellery in both an artistic and scientific context. Exploration and analysis of smart materials that are suitable for use in a jewellery context form an important part of this research, and the consequent summary of these findings in a comprehensive publication and through research exposition will aid craft practitioners in developing a meaningful discourse within the field by encouraging further experimentation and critical debate about the suitable aesthetic integration of smart materials within a jewellery context. Recognising the potential for these new materials and advanced technologies to be included in the repertoire of artistic self-expression will enable the discipline of contemporary jewellery to move forward by maintaining its relevance in this digital age and to enhance its contribution to contemporary culture in a wider sense.

\section{Artistic Practice and Material} Innovation. The symbiotic relationship and interaction between the body and a stimulusresponsive jewellery object, is a concept that has continuously fuelled my own practice as a studio jewellery artist and craft practitioner. Initially, this interest was expressed through a body of work that utilises experimentation with alternative materials, most notably silicone rubber (Fig.3), to develop components that facilitate the visual mutation of an artificial jewellery organism into a natural extension of the body by mimicking processes of movement and growth observed in the marine environment. The notion of creating a growing, living jewellery organism to interact with the wearer's body continues to inform my practice, as does the concept of biomimicry. An investigation of the harmoniously imperfect intricacies of structural growth patterns found in nature and their abstracted counterparts in modern architecture, has led me to develop an aesthetic that uses these structural components to create jewellery that emerges from within the complex geometries of the human body. To further this line of enquiry I am exploring the development of jewellery objects that visually propagate upon the wearer and react to physiological changes or environmental stimuli.

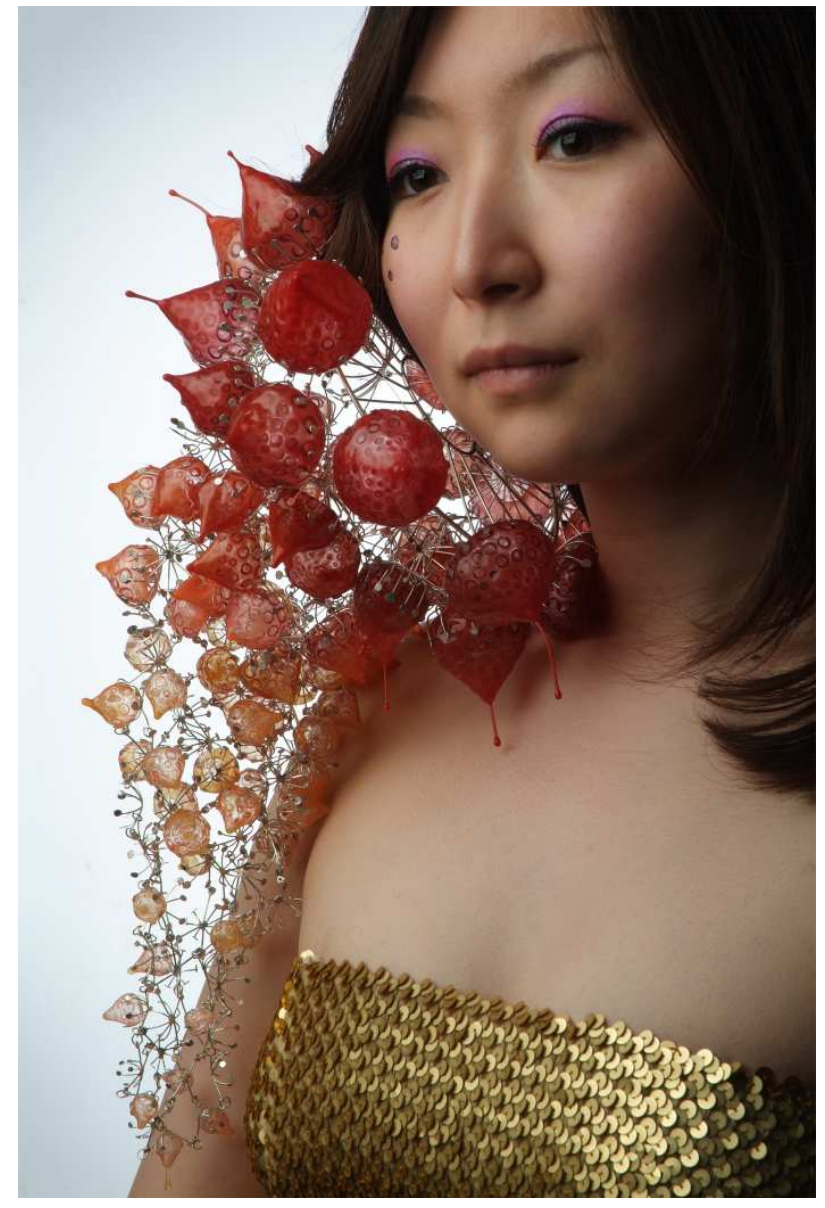

Fig.3: Earconch, Katharina Vones 2006, Sterling Silver, Silicone 
Collaborative Strategies The initial stage of this research project focuses on exploring a range of stimulus-reactive smart materials, and testing their usability in terms of creating wearable objects that react directly and intimately to the body and other environmental stimuli. The selection of and experimentation with suitable materials constitutes a significant part of this practice-led research. I am currently looking for materials scientists, researchers and developers who could contribute their expertise and would like to undertake a collaborative project with me. A thorough evaluation of selected materials through a specified experimental protocol - which could serve to analyse factors such as physical and mechanical properties, availability, technical feasibility, usability in a jewellery context, health and safety implications and



Fig.4: Triangulitis Ring, Katharina Vones 2010, Sterling Silver, Aquamarine aesthetic appeal - is the first step of such a collaborative project. Once a range of suitable materials for use in jewellery objects has been selected, the research will move to its next stage of exploring the integration of wearable technologies within these objects. Many smart materials need the stimulus provided by the body to be translated through a sensor placed within an electronic component in order to exhibit their particular reactive properties. The practical skills available to the contemporary jeweller facilitate such collaboration, and the contribution of a different point of view might yield unexpected results. As an example, the method of photo-etching and subsequently folding sterling silver sheet metal into hollow three dimensional structures, which comprises a key element of my most recent body of work (Fig.4), could easily be adapted to include internal electrical circuitry. Laserwelding and rapid-prototyping are other techniques that lend themselves to the development and manufacture of miniaturised electronic components as well as their unobtrusive integration into jewellery objects. The creation of computerised jewellery organisms that are capable of mimicking processes found in nature such as accelerated growth and phototropism is one possible outcome of this research.

Looking towards the Future Potential practical applications of stimulus-reactive jewellery exist in the areas of human computer interaction, transplant technology, identity management and artificial body modification including prosthetics, where such symbiotic jewellery organisms could be used to develop visually engaging yet multi-functional enhancements of the human body. Benefactors of these advances are those with conditions that need the support of extraneous devices, currently so often disguised rather than celebrated as enhancements of the human form in their own right.

\section{References}

[1] P. Lichty, Building a culture of ubiquity, Intelligent Agent Magazine, Vol.8.1 (2008), retrieved from http://www.intelligentagent.com

[2] J. Wallace, M. Press, All this useless beauty: the case for craft practice in design for a digital age, The Design Journal , 7 (2004) 42-53.

[3] J. Wallace, Emotionally charged: a practice-centred enquiry of digital jewellery and personal emotional significance, retrieved from http://www.digitaljewellery.com/ 
[4] J. Wallace, D. Jackson, C. Ladha, P.Olivier, A. Monk, M. Blythe, P. Wright, Digital jewellery and family relationships, retrieved from

http://homepages.cs.ncl.ac.uk/p.l.olivier/Publications_files/OlivierFACTa07.pdf

[5] S. Kettley, Framing the ambiguous wearable, Convivio Web-Zine (2005), retrieved from http://daisy.cti.gr/webzine/Issues/Issue\%202/Articles/Framing\%20the\%20ambiguous\%20wearable/ index.html

[6] S. Kettley, Crafting the wearable computer - design processes and user experience, retrieved from http:/www.scribd.com/doc/5146612/Crafting-the-Wearable-Computer-design-process-anduser-experience-thesis-Vol-1

[7] S. Kettley, Crafts praxis for critical wearables Design, Proceedings Wearable Futures Conference, University of Wales, Newport (2005). Also in AI \& Society Vol. 22 (2007) 5-14.

[8] D. Humphrey, Strategies for engaging with digital technologies in the development of future jewellery, Presentation given at the Methods Network Seminar Blue Skies and Singing Rings: Digital Technologies and Jewellery of the Future (2006), retrieved from http://www.artshumanities.net/node/1182

[9] L. Williams, Technology, jewellery and medicine, Presentation given at the Methods Network Seminar Blue Skies and Singing Rings: Digital Technologies and Jewellery of the Future, retrieved from http://www.arts-humanities.net/node/1183

[10] P. Wright, J. Wallace, J. McCarthy, Aesthetics and experience-centred design, ACM Trans. Comput.-Hum. Interact. 15, 4 (2008) Article 18

[11] S. Kettley, Visualising social space with networked jewellery, retrieved from http://www.sarahkettleydesign.co.uk/sarahkettley/publications_sarah_kettley_files/Visualising\%2 0Social\%20Space\%20with\%20Networked\%20Jewellery.pdf

[12] T. Saburi, Ti-Ni shape memory alloys, in K. Otsuka, C.M Wayman (Eds.), Shape memory materials, Cambridge University Press, Cambridge, 1998, pp.49-97.

[13] M. Di Ventra, Y.V Pershin, Memory materials: a unifying description, Mater Today, Vol. 14, (2011) 584-591.

[14] M. Behl, A. Lendlein, Shape-memory polymers, Mater Today, Vol.10, (2007) 20-28

[15] T. Ikeda, T. Ube, Photomobile polymer materials: from nano to macro, Mater Today, Vol. 14, (2011) 480-487.

[16] K. Uchino, Shape memory ceramics, in K. Otsuka, C.M Wayman, (Eds.), Shape memory materials, Cambridge University Press, Cambridge, 1998, pp.184-203.

[17] N. Cherry, Grow your own - angiogenetic body adornment, in SCAN Journal of Media Arts and Culture, Vol. 3 No. 3 (2006)

[18] For further information http://www.biojewellery.com/

[19] M. Miodownik, The case for teaching the arts, Mater Today, Vol.6 (2003) pp. 36-42. 
Next Generation Micro/Nano Systems

10.4028/www.scientific.net/AST.81

Foreign Bodies - The Creation of Symbiotic Jewellery through the Development and Application of Stimulus-Responsive Smart Materials and Microelectromechanical Systems

10.4028/www.scientific.net/AST.81.49 